\title{
Über die Behandlung der Tollwut mit Kantharidin.
}

\author{
Von Prof. Dr. Fritz Netolitzky.
}

In Europa, Asien und Afrika behandelt man die von tollen Hunden Gebissenen auch heute noch mit Insektenpräparaten, die kantharidinhaltig sind. Diese Übung ist zunächst ganz unverständlich, wenn wir uns an den internen Gebrauch klammern, der anscheinend heute vorherrscht. Als Beispiel sei aus der „Vergleichenden Volksmedizin“ von Hovorka und Kronfeld, Bd. II, 432, erwähnt :

„Seit langer Zeit besitzen die Araber in Südtunesien ein Mittel gegen die Hundswut, und zwar sind es die Nachkommen des Sidi Ali Nuri, welche die Bereitung dieser Arznei und die Verabreichung übernommen haben. Sie bereiten aus einer hier vorkommenden Kantharidenart, der sie Hinterleib, Flügel und Füße wegnehmen, einen Trank, den der Gebissene in einer bestimmten Anzahl von Tagen zu sich nehmen muß. Es stellt sich dann Blutharnen ein. Im Blutgerinnsel sieht der Glaube kleine Hunde, die ausgeschieden werden, und damit ist der Kranke gesund . . . Die Araber benützen ein Mittel aus Meloë tuccius Rossi und Mylabris tenebrosa Castelnau innerlich; es darf nur ein Stück von Weizenkorngröße in ungesalzener Fleischsuppe innerhalb der ersten zwanzig Tage nach erfolgtem Bisse genommen werden."

Eine Erklärung für diese Mißhandlung des Kranken zur Verhütung des Ausbruches der Tollwut könnte höchstens in dem Satze gipfeln „Gleiches mit Gleichem behandeln“, jenes Schlagwort („similia similibus"), das auch heute noch nicht überwunden ist. Denn auf der Höhe der Vergiftung mit Kantharidinträgern ähnelt das Bild doch immerhin der Rabies so weit, daß man diesen Gedanken nicht ganz beiseite lassen kann.

Aus der älteren Literatur geht aber ziemlich ungezwungen hervor, daß wenigstens an manchen Orten nur die lokale Behandlung des Hundebisses mit Kantharidin geübt wurde, was natürlich die ganze Sache in ein anderes Licht. setzt. So berichtet ein gewisser Moufet ${ }^{1}$ ), ein Schüler Ge $3 \mathrm{n}$ ers, der dessen Naturgeschichte um den Band „Insekten" vermehrt hat, folgendes (p. 163):

1) Moufet, Insectorum vel minimorum animalium theatrum 1634 . 
„Casparus Reglerus (liber de peste) rät 20 Maiwürmer zu sammeln, und zwar nicht mit den Fingern, sondern mit zwei Stäbchen; hat man sie dann in einen irdenen Topf oder ein Glas gegeben, so erstickt man sie in feinstem Olivenöl. Mit diesem Öle reibt man Bubonen, Karbunkel, Pestgeschwüre und Wundbisse von tollen Hunden ein und heilt sie dadurch . . . weil sie in wunderbarer Weise das Gift ausziehen und die betreffende Stelle rasch zur natürlichen Temperatur zurückbringen."

Nach dieser Beschreibung ist es ganz klar, daß hier die äußerliche Anwendung, die Ätzwirkung, das Wesentliche ist. Lapisstifte und andere unserer energischen Ätzmittel, z. B. Mineralsäuren, hatte das Volk nicht und Glüheisen und Messer waren noch gefürchteter als heutzutage. Ganz besonders wichtig ist die äuferliche Behandlung des Bisses toller Hunde mit Kantharidin. Ein solcher Biß durchdringt nämlich häufig gar nicht die Haut; es handelt sich in vielen Fällen mehr um Zahnabdrücke als um blutende Wunden. Und doch kann die Tollwut ausbrechen. Werden nun solche Stellen sofort nach dem Bisse mit Meloesaft, Kantharidin usw. bestrichen, so ist dies mindestens so viel wert, als wenn der Gebissene viele Stunden später vom Arzte mit Lapis geätzt würde. Das Katharidin zerstört rasch die lebenden Zellen und dadurch könnte tatsächlich das noch nicht in den Kreislauf gelangte Wutgift wirkungslos gemacht werden.

Ich halte diese externe Anwendung als Ätzmittel bei Hundebissen für das ursprüngliche Verfahren, gleichgültig ob hiezu Meloë, Lytta oder Mylabris dienen oder ob Europa, Nordamerika oder China in Betracht kommen.

Verfolgen wir die Literatur weiter, so finden wir Vorschriften, bei denen die äußerliche Verätzung des Hundebisses mit der innerlichen Anwendung verquickt sein kann, wobei uns wieder die Volksanwendung leitet:

„Der Maiwurm (Meloë) wurde seit dem Jahre 1777 in Preußen als spezifisches Mittel gegen Hundswut empfohlen, und zwar nach folgender Vorschrift: Den Maiwurm greife man nicht mit der Hand, sondern mit Papier an, tue ihn in Honig und laß ihn darin stecken; von dem Honig gibt man in warmes Bier und salbe auch die Wunde, die ein toller Hund gebissen hat, damit, so wird er 'gesund... Gegen die Hundswut lege man nach Vorschriften in Westböhmen einen Teig aus dem Pulver der Schmalzkuh (Meloë), Petersilie und (Melassen-) Syrup auf die Wunde und nehme aus letzterem gemachte Kügelchen ein ... und gebe innerliche Abkochungen von spanischen Fliegen." (Hovorka \& Kronfeld [p. 424-425].) 
Diese Art der Wutbehandlung wurde von Ärzten und Laien fleißig geübt, ja sogar kommissionell geprüft: Es glaubte ein österreichischer Grundbesitzer so fest an die Wirksamkeit der Maiwürmer in Olivenöl, daß das Mittel auf seine Anregung hin amtlich geprüft und durch eine Verkettung günstiger Umstände auch als wirksam erkannt wurde. Die Regierung verlieh ihm für die Bekanntgabe des Mittels eine Rente und den Adel mit dem Prädikate „Edler von Wuthwehr". (Abels, Grof' Archiv 1912, l. c., p. 230.)

Und die vermeintliche Heilung.? Eigentlich geht aus der Anwendungsweise hervor, daf man mit Maiwürmern oder Kanthariden den Ausbruch der Krankheit verhindern wollte und nicht etwa die schon ausgebrochenen Symptome bekämpfte. Und da bei weitem nicht alle von wirklich wutkranken Hunden Gebissene an Rabies erkranken, so ist die Täuschung über den Heilwert dem Volke und den überzeugungstreuen Ärzten nicht allzusehr übelzunehmen.

Auch nach anderen Richtungen läßt sich der Gebrauch des Kantharidins als Ätzmittel beim Volke führen. So wird Meloësaft ins Ohr getropft, um schwere Zerstörungen herbeizuführen (Militärbefreiungsschwindel). Chronische Hautkrankheiten werden energisch damit geätzt, selbst Krebsgeschwïre suchte man damit auszurotten und ebenso die Lepra. Man entfernte Warzen und eingewachsene Nägel. Wieder schreibt Moufet bierüber: „Kanthariden mit Kalk gemischt entfernen die Haut wie mit dem Messer... man hüte sich aber vor zu ausgiebigen Exulzerationen, gegen die man Galle, Mäusekot mit Helleborus und Pfeffer anwendet." Übrigens haben die alten Ärzte, z. B. Hippokrates, Arzneibereitungen aus diesen Käfern fast nur extern angewendet, sogar bei Uteruserkrankungen, verhaltener Periode, Wehenschwäche und zur Fruchtabtreibung.

Die interne Verwendung von Kantharidinträgern, besonders zu Geschlechtsreizungen, ist in Mitteleuropa überhaupt relativ jung; so wurde die einheimische Meloë fast nicht als "Liebesmittel" gebraucht, sondern es spielten bei uns die Am eis en eine, wenn auch schwächliche und minder gefährliche Rolle. Erst von Westeuropa gelangte der Mißbrauch der spanischen Fliege als Geschlechtsstimulanz zu uns.

Einige Worte über die relative Immunität des Igels gegen Kantharidin mögen hier noch Platz finden, da diese Eigenschaft vielfach untersucht ist, meist aber als etwas ganz einzig Dastehendes betrachtet wird. Nun sind aber noch andere Tiere relativ immun gegen das Gift: Frosch, Ente, Huhn, Schwalbe und Fledermaus. 
Offenbar muß auch der Kuckuck gegen das äußerst stark reizende Prinzip in den Raupen des Prozessionsspinners gefeit sein, da er sie massenhaft vertilgt.

Meine Untersuchungen (Pharm. Post 1916, „Insekten als Heilmittel“) zeigen, daß diese relative Immunität eine allgemeine Eigenschaft aller Insektenfresser sein dürfte.

Dadurch wird freilich keine Erklärung gegeben; die Frage wird nur auf eine breitere Basis gestellt, da die scheinbaren Ausnahmen zu einer Regel erhoben werden können.

Lederia Seidlitzi n. sp. (Col. Melandryidae.)

Von Edm. Reitter in Paskau.

Durch den nur hinten deutlich gerandeten und gekanteten Halsschild mit anatolica Friv. zunächst verwandt, aber viel kleiner, zarter und durch die äußerst feine, ziemlich weitläufige Punktur der Oberseite leicht zu unterscheiden. Von der kaukasischen L. suramensis Reitt., außer der unvollständigen Kantung der Halsschildseiten durch schmälere, länglicher elliptische Körperform, die an den Seiten des Halsschildes nicht ausgebuchtete Basis und die nicht spitzigen, sondern höchstens rechteckigen Hinterwinkel abweichend.

Elliptisch, hoch gewölbt, rotgelb, sehr fein und kurz, anliegend gelblich behaart. Die Fühler erreichen die Basis des Halsschildes. Kopf und Halsschild mit äußerst feiner, schwer wahrnehmbarer, undichter Punktur, die Seiten des queren Halsschildes nach vorne verengt, nach vorne wenig über die Mitte gekantet und fein gerandet, die Basis gerade, davor mit angedeuteter, sehr seichter und feiner, an den Seiten erloschener Querlinie, die Hinterwinkel fast rechteckig oder wenig stumpf, die Vorderwinkel niedergebogen. Schildchen dreieckig, nur punktförmig. Flügeldecken mit sehr feiner, wenig dichter Punktur. Körper der L. suramensis wegen der Punktur der Oberseite ähnlicher, aber doch der anatolica, wegen der unvollständigen Råndung des Halsschildes näher verwandt. L. $1.8 \mathrm{~mm}$.

Kleinasien: Sabandja.

Herrn Dr. G. von Seidlitz, der die verwandten Genera von Lederia richtig stabilisiert hat, gewidmet. 


\section{$2 \mathrm{BHL}$ Biodiversity Heritage Library}

Netolitzky, Fritz. 1916. "Über die Behandlung der Tollwut mit Kantharidin." Wiener entomologische Zeitung 35, 287-290.

https://doi.org/10.5962/bhl.part.10000.

View This Item Online: https://www.biodiversitylibrary.org/item/45303

DOI: https://doi.org/10.5962/bhl.part.10000

Permalink: https://www.biodiversitylibrary.org/partpdf/10000

\section{Holding Institution}

Smithsonian Libraries

\section{Sponsored by}

Smithsonian

\section{Copyright \& Reuse}

Copyright Status: NOT_IN_COPYRIGHT

This document was created from content at the Biodiversity Heritage Library, the world's largest open access digital library for biodiversity literature and archives. Visit BHL at https://www.biodiversitylibrary.org. 